\title{
Pippard Relations Close to the Melting Point in the Premelting Region of Hexadecane
}

\author{
Hamit Yurtseven, ${ }^{1}$ Özcan Tilki, ${ }^{1}$ and Mustafa Kurt ${ }^{2}$ \\ ${ }^{1}$ Department of Physics, Middle East Technical University, 06531 Ankara, Turkey \\ ${ }^{2}$ Department of Physics, Çanakkale 18 Mart University, 17100 Çanakkale, Turkey \\ Correspondence should be addressed to Hamit Yurtseven, hamit@metu.edu.tr
}

Received 6 August 2012; Accepted 15 October 2012

Academic Editor: Dominique Richon

Copyright (C) 2012 Hamit Yurtseven et al. This is an open access article distributed under the Creative Commons Attribution License, which permits unrestricted use, distribution, and reproduction in any medium, provided the original work is properly cited.

\begin{abstract}
We examine here the validity of the Pippard relations close to the melting point in the premelting region of hexadecane. For this verification, we analyze the observed data for the thermal expansivity $\alpha_{p}$ obtained for various pressures at constant temperatures of 302 and $325 \mathrm{~K}$ in this system. By calculating the isothermal compressibility $\kappa_{T}$ and the specific heat $C_{p}$ in the same pressure region of the premelting region of hexadecane, we obtain that $C_{p}$ varies linearly with $\alpha_{p}$ and also that $\alpha_{p}$ varies linearly with $\kappa_{T}$ for this system. This indicat es that some molecular organic compounds, such as solid hexadecane studied here, can exhibit $\lambda$-phase transitions prior to melting which are expected to verify the Pippard relations.
\end{abstract}

\section{Introduction}

Phase behaviour of pure n-alkanes is important for industrial and scientific applications. The controlled precipitation of solid phases of these mixtures is widely applied in pharmaceutical, cosmetic, and food industries as a separation process, as also pointed out previously [1]. Those paraffin waxes are also used in latent thermal energy storage applications, and additionally for cool storage applications in regard to the phase transition processes a binary mixture of tetradecane and hexadecane is widely used [2].

In the literature, various sources of experimental data have been reported in regard to the thermodynamic properties of hexadecane. Some recent experimental reports are the following: $T-X$ phase diagrams [1], entropy changes with molality at constant temperature [3], an anomalous heat effect during colloidal phase transitions [4], determination of the melting temperature $\left(6.8^{\circ} \mathrm{C}\right)$ and a corresponding heat of fusion $(121 \mathrm{~kJ} / \mathrm{kg})$ for a mixture of tetradecane $(40 \%)$ and hexadecane $(60 \%)$ [2], and also the crystallization temperatures of two binary mixtures: the tetradecane + hexadecane and tetradecane + pentadecane system up to $100 \mathrm{MPa}$ [1].

Solid hexadecane among long-chain paraffin solids exhibits considerable precursor effects [5]. It has been shown that there exists an orientational disorder around the long axis of the molecule in solid hexadecane [6]. Experimentally, it has been demonstrated that this is a kinked chain-like disorder rather than a librational disorder $[7,8]$, as also indicated by Pruzan et al. [9]. This orientational disorder occurring in solid hexadecane gives rise to instability and melting of the solid phase. Instability and melting have been associated with a soft phonon of zero wave vector [10-13].

Solid hexadecane melts into the liquid phase as a second-order transition associated with a particular type of orientational disorder which depends on the symmetry [9]. As in many molecular organic compounds, solid hexadecane exhibits orientational disorder in a small range where the thermodynamic quantities such as the thermal expansivity $\alpha_{p}$, isothermal compressibility $\kappa_{T}$, and the specific heat $C_{p}$ show anomalous behaviour close to the melting point $[5,9]$.

The anomalous behaviour of $\alpha_{p}, \kappa_{T}$, and $C_{p}$ can be described by a power-law formula near the melting point in solid hexadecane. In the premelting region of hexadecane close to the melting point, we can assume that $\alpha_{p}, \kappa_{T}$, and $C_{p}$ exhibit similar critical behaviour associated with the same critical exponent. Buckingham and Fairbank [14] have indicated that $C_{p}$ behaves like $\kappa_{T}$ and $\alpha_{p}$ in the vicinity of the critical point. In the precursor zone of the solid hexadecane, $C_{p}, \alpha_{p}$, and $\kappa_{T}$ are expected to increase as approaching 
the melting point. This increase in the thermodynamic quantities considered is similar to the critical behaviour of $\lambda$ phase transitions. Pippard has established a linear variation of the specific heat $C_{p}$ with the thermal expansivity $\alpha_{p}$ in the vicinity of the $\lambda$-phase transition in $\mathrm{NH}_{4} \mathrm{Cl}$ [15]. The Pippard relations have been tested for a variety of materials exhibiting $\lambda$-phase transitions.

In this study, we also examine the Pippard relations in the premelting region of hexadecane near the melting point. For this examination, we calculate the isothermal compressibility $\kappa_{T}$ and the specific heat $C_{p}$ from our analysis of the thermal expansivity $\alpha_{p}$ which was measured experimentally at various pressures near the melting point for constant temperatures of 302 and $325 \mathrm{~K}$ in solid hexadecane [9]. By obtaining the values of the critical exponent $\gamma$ from our analysis, we calculate $\kappa_{T}$ and $C_{p}$ as functions of pressure in the premelting region of hexadecane. We then establish a linear variation of $C_{p}$ with $\alpha_{p}$ (first Pippard relation) and also a linear variation of $\alpha_{p}$ with $\kappa_{T}$ (second Pippard relation) close to the melting point in this molecular organic compound.

In Section 2, we review the method and conditions about experimental study of Pruzan et al. [9]. In Section 3, our calculations and results are presented. In Sections 4 and 5, we present our discussion and conclusions, respectively.

\section{Review of the Experimental Technique}

Using a $P-V-T$ method, the thermal expansivity $\alpha_{p}$ was observed by Pruzan et al. [9] at various pressures close to the melting point. Using a high-pressure calorimetric method, the heat of compression was measured. The measurement of heat, in open-system conditions, was conducted, which flows out of (into) the sample when it is compressed (decompressed) isothermally [9]. The thermal expansivity $\alpha_{p}$ was then determined according to the first Maxwell equation:

$$
\alpha_{p}=-V^{-1}\left(\frac{\partial S}{\partial T}\right)_{P}
$$

where $V$ and $S$ are the molar volume and entropy of the sample, respectively. This technique was referred to as the piezothermal method [9]. For the measurements, the pressure steps were about $20 \mathrm{MPa}$ far from the melting point and they were $0.1-10 \mathrm{MPa}$ close to the melting point [9]. For their measurements, Pruzan et al. [9] reported that their observations were made over different times, sometimes greater than $72 \mathrm{~h}$, during which no change could be observed in the expansivity variation against pressure for hexadecane.

\section{Calculations and Results}

Near the melting point of solid hexadecane, the thermodynamic quantities such as the thermal expansivity $\alpha_{p}$, isothermal compressibility $\kappa_{T}$, and the specific heat $C_{p}$ can exhibit critical behaviour. This critical behaviour of the thermal expansivity can be expressed as

$$
\alpha_{p}=A\left(P-P_{m}\right)^{-\gamma},
$$

where $\gamma$ is the critical exponent for $\alpha_{p}$ and $A$ is the amplitude. Here, $P_{m}$ denotes the melting pressure of solid hexadecane, which is close to the critical point [5]. Using the thermodynamic relation near the melting point:

$$
\frac{\alpha_{p}}{\kappa_{T}}=\left(\frac{d P_{m}}{d T}\right),
$$

the isothermal compressibility $\kappa_{T}$ can be expressed as a function of pressure

$$
\kappa_{T}=\frac{A\left(P-P_{m}\right)^{-\gamma}}{\left(d P_{m} / d T\right)}
$$

by means of (2).

We can also obtain the pressure dependence of the specific heat $C_{p}$ using the thermodynamic relation near the melting point as follows:

$$
C_{p}=T V \alpha_{p}\left(\frac{d P_{m}}{d T}\right) .
$$

In (5), the volume $V$ is a function of pressure, which can be obtained as

$$
V_{T}(P)=V_{m} \exp \left[\frac{A}{1-\gamma} \frac{1}{\left(d P_{m} / d T\right)}\left(P-P_{m}\right)^{1-\gamma}\right],
$$

where $V_{m}$ denotes the melting volume of solid hexadecane. Equation (6) can be derived easily using the definition of the isothermal compressibility $\kappa_{T}=-(1 / V) /(\partial V / \partial P)_{T}$. Thus, the pressure dependence of the molar volume $V_{T}(P)$ has been derived here, as given by (6), in the case when the isothermal compressibility $\kappa_{T}$ is a function of pressure according to (4). We note here that when the isothermal compressibility $\kappa_{T}$ is a function of the molar volume $V$, the pressure dependence of the molar volume $V_{T}(P)$ can also be derived. But, its functional form should not be the same as (6) since the volume dependence of the isothermal compressibility $\kappa_{T}$ changes the pressure dependence of the molar volume in the premelting region close to the melting point of solid hexadecane. Finally, by means of (5), the pressure dependence of the specific heat $C_{p}$ can be written as a power-law formula:

$$
C_{p}=A T V\left(\frac{d P_{m}}{d T}\right)\left(P-P_{m}\right)^{-\gamma} .
$$

In the vicinity of the melting point of solid hexadecane, the specific heat $C_{p}$ can be related to the thermal expansivity $\alpha_{p}$ linearly, according to the first Pippard relation:

$$
C_{p}=T V\left(\frac{d P_{m}}{d T}\right) \alpha_{p}+T\left(\frac{d S}{d T}\right)_{m} .
$$

Similarly, the thermal expansivity $\alpha_{p}$ can be related linearly with the isothermal compressibility $\kappa_{T}$ by the relation in the vicinity of the melting point:

$$
\alpha_{p}=\left(\frac{d P_{m}}{d T}\right) \kappa_{T}+\frac{1}{V}\left(\frac{d V}{d T}\right)_{m}
$$


according to the second Pippard relation. Thus, linear plots of $C_{p}$ against $\alpha_{p}(8)$ and $\alpha_{p}$ against $\kappa_{T}$ (9) can be obtained near the melting point in solid hexadecane. From those linear plots, (8) and (9), we can extract the values of the slope $d P_{m} / d T$ numerically for solid hexadecane close to the melting point.

We reanalyzed here the thermal expansivity $\alpha_{p}$ measured at various pressures in the premelting region close to the melting point for constant temperatures of 302 and $325 \mathrm{~K}$ in solid hexadecane due to Pruzan et al. who also analyzed their experimental data [9]. For our analysis, we followed the steps, as given below.

(1) We used the power-law formula (2) in the logarithmic form obtained as

$$
\ln \alpha_{p}=\ln A-\gamma \ln \left(P-P_{m}\right)
$$

for the premelting region of hexadecane. We then extracted the values of the critical exponent $\gamma$ as $2.06 \pm 0.05$ and $1.97 \pm 0.04$ with the values of the amplitude $A$ for constant temperatures of 302 and $325 \mathrm{~K}$, respectively, as given in Table 1 . Similarly, from their analysis of the thermal expansivity $\alpha_{p}$, Pruzan et al. [9] have obtained the value of the critical exponent as $\gamma_{m}=1.9$ in the melting zone of hexadecane. Figure 1 gives the thermal expansion $\alpha_{p}$ plotted as a function of $P-P_{L}$ in a log-log scale for constant temperatures of $302 \mathrm{~K}$ and $325 \mathrm{~K}$. Since we used for our analysis the experimental data in the melting zone, which was obtained in the range $P-P_{L}<50 \mathrm{MPa}$ where $P_{L}$ is the liquid or melting $\left(P_{m}\right)$ pressure [9], we were able to describe the critical behaviour of the thermal expansivity $\alpha_{p}$ in the premelting region of hexadecane according to (10). On the other hand, $P-P_{L}>50 \mathrm{MPa}$ corresponds to the precursor zone (solid phase) for hexadecane [9]. These pressure ranges have also been reported in the work of Pruzan et al. [9] as $P_{1}-P_{L}=140 \mathrm{MPa}$ and $P_{2}-P_{L}=60 \mathrm{MPa}$, where $P_{1}$ represents the beginning of the precursor range and $P_{2}$ is the pressure for a change of regime, which is closer to the critical pressure $P_{C}$ for the solid hexadecane.

(2) Using those values of $\gamma$ and $A$ which we obtained (Table 1), we then calculated the isothermal compressibility $\kappa_{T}$ as a function of pressure according to (4) where we used the experimental value of $d P_{m} / d T=10 \mathrm{MPa} / \mathrm{K}[9]$.

(3) The next step was to calculate the pressure dependence of the volume $V_{T}(P)$ in the premelting region of hexadecane near the melting point for constant temperatures of 302 and $325 \mathrm{~K}$ using (6). In this equation, the values of the melting volume $V_{m}$ [16], as given in Table 1, were used with the experimental value of $d P_{m} / d T=10 \mathrm{MPa} / \mathrm{K}$.

(4) Finally, the pressure dependence of the specific heat $C_{p}$ was calculated by (7) for the premelting region of hexadecane near the melting point for those constant
TABle 1: Values of $\gamma$ and $A$ extracted (Figure 1) for $\alpha_{p}$ using the experimental data [9] and the $V_{m}$ values for the solid hexadecane at temperatures indicated.

\begin{tabular}{lccc}
\hline$T(\mathrm{~K})$ & $\gamma$ & $A(\mathrm{MPa})^{\gamma} / \mathrm{K}$ & $V_{m} \times 10^{-6}\left(\mathrm{~m}^{3} / \mathrm{mol}\right)$ \\
\hline 302 & $2.06 \pm 0.05$ & 1.9837 & 294.93 at $303 \mathrm{~K}$ \\
325 & $1.97 \pm 0.04$ & 1.3624 & 300.13 at $323 \mathrm{~K}$ \\
\hline
\end{tabular}

temperatures considered using $V_{T}(P)$ values (6) with the $d P_{m} / d T$ value [5] given above.

Since we obtained the pressure dependence of the thermal expansivity $\alpha_{p}$ (2), isothermal compressibility $\kappa_{T}(4)$, and the specific heat $C_{p}(7)$ in the same pressure interval for constant temperatures of 302 and $325 \mathrm{~K}$ in the premelting region of hexadecane, we were then able to establish the Pippard relations ((8) and (9)) in this crystalline system. We plot the specific heat $C_{p}$ against the thermal expansivity $\alpha_{p}$ in the premelting region of hexadecane near the melting point for constant temperatures of $302 \mathrm{~K}$ and $325 \mathrm{~K}$ in Figure 2.

We also examined the second Pippard relation (9) by $\alpha_{p}$ as a function of $\kappa_{T}$ in the premelting region of hexadecane close to the melting point. Figure 3 gives our plot for constant temperatures of 302 and $325 \mathrm{~K}$. From our plots of $C_{p}$ against $\alpha_{p}$ (first Pippard relation) and $\alpha_{p}$ against $\kappa_{T}$ (second Pippard relation), we extracted the value of the slope as $d P_{m} / d T=$ $10 \mathrm{MPa} / \mathrm{K}$. Since the experimental value of $d P_{m} / d T=$ $10 \mathrm{MPa} / \mathrm{K}[5]$ was used as the initial value in (4) (step 2), (6) (step 3), and (7) (step 4) to calculate $\kappa_{T}, V_{T}(P)$, and $C_{P}$, respectively, as a function of pressure, we should expect the same slope value of $10 \mathrm{MPa} / \mathrm{K}$ from (8) as well.

\section{Discussion}

We examined here whether the specific heat $C_{p}$ varies linearly with the thermal expansivity $\alpha_{p}$ (first Pippard relation) and also $\alpha_{p}$ varies linearly with the isothermal compressibility $\kappa_{T}$ (second Pippard relation) in the vicinity of the melting point in the premelting region of hexadecane. As seen from our plots (Figures 2 and 3), we established the Pippard relations ((8) and (9)) with the slope $d P_{m} / d T=10 \mathrm{MPa} / \mathrm{K}$ which was extracted for this system. This indicates that those thermodynamic quantities, namely, $C_{p}, \alpha_{p}$, and $\kappa_{T}$ exhibit similar critical behaviour close to the melting point in the premelting region of hexadecane.

From our analysis of the measured thermal expansivity, we extracted the value of $\gamma=2$ as the critical exponent for constant temperatures ( $302 \mathrm{~K}$ and $325 \mathrm{~K}$ ), in the premelting region of hexadecane (Table 1). Our exponent value is very close to the value with the uncertainties (Table 1) of $\gamma=$ 1.9 as obtained by Pruzan et al. [9]. They analyzed their experimental data according to the power-law relation (2) with the $P_{m}=P_{C}$. The critical pressure $P_{C}$ at which $\alpha_{p}$ diverges due to the precursor transformation is always between $P_{2}$ and $P_{L}$ [9]. For hexadecane, $P_{C}$ is close to $P_{2}$, as indicated previously. Within the pressure ranges $P_{1}-P_{L}=$ $140 \mathrm{MPa}$ and $P_{2}-P_{L}=60 \mathrm{MPa}$, Pruzan et al. [9] obtained the values of $\gamma_{S}=0.5$ in the solid phase, $\gamma_{P}=0.7$ in the precursor zone, and $\gamma_{m}=1.9$ in the melting zone, where the pressure 


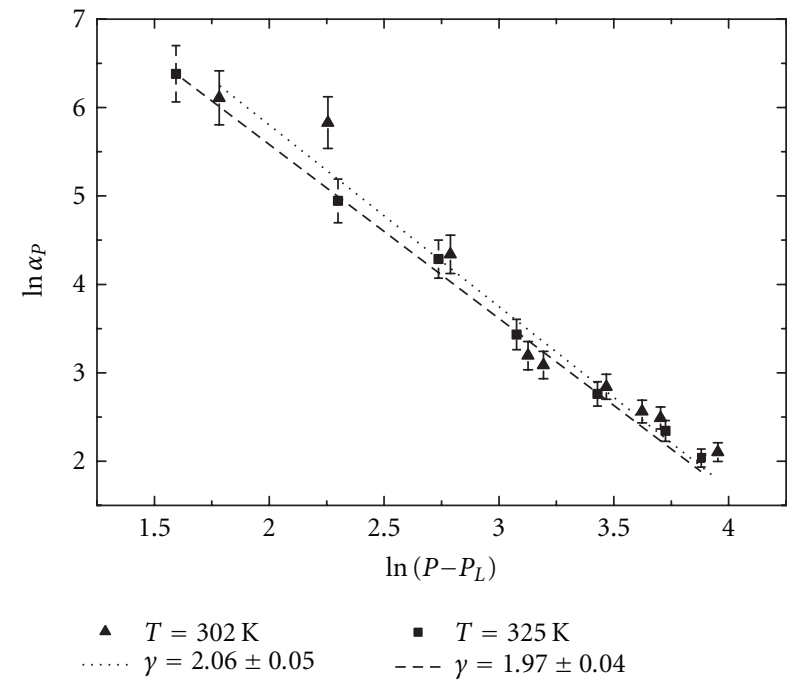

Figure 1: $\alpha_{p}$ versus $P$ in a $\log$-log scale close to $P_{m}$ in the premelting region of hexadecane for $T=302$ and $325 \mathrm{~K}$ (10) when $P_{m}=P_{L}$ [9]. The experimental data [9] with the uncertainties extracted (error bars) are also given here.

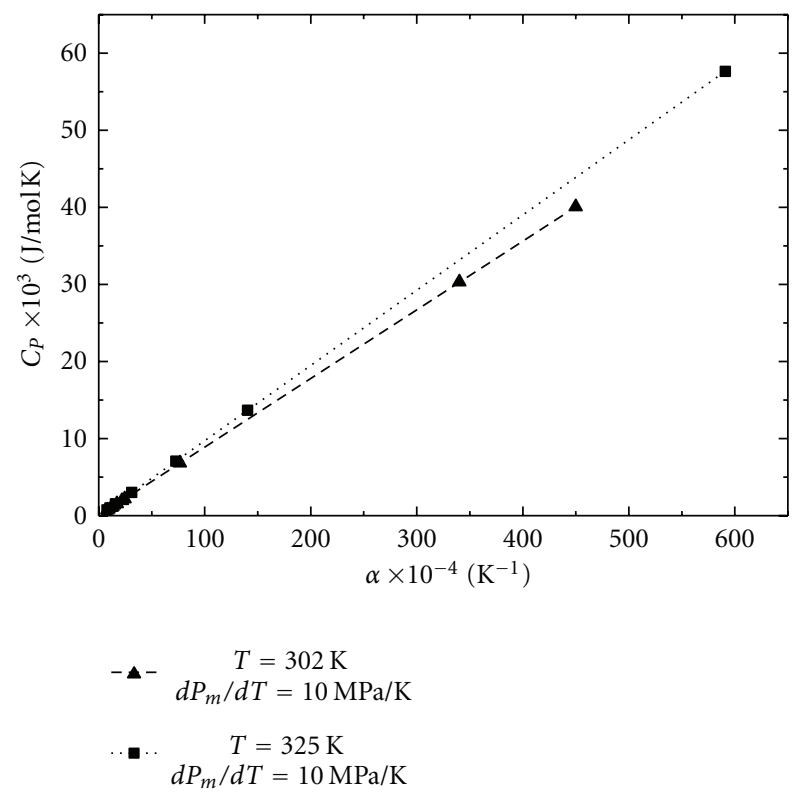

Figure 2: $C_{p}$ versus $\alpha_{p}$ at various $P$ close to $P_{m}$ for $T=302$ and $325 \mathrm{~K}$ in the premelting region of hexadecane (8). Observed $\alpha_{p}$ values [9] are shown here.

range is greater than the melting pressure $P_{m}$. The exponent value of 1.9 or 2 from both analyses in the melting zone can be compared with an effective exponent greater than 1 for the critical behaviour of the thermal expansivity $\alpha_{p}$ due to surface melting model of Lipowsky and Speth $[17,18]$, as also pointed out previously [9]. It has been interpreted that the second regime (liquid phase) observed in molecular organic compounds might be ascribed to impurity effects for

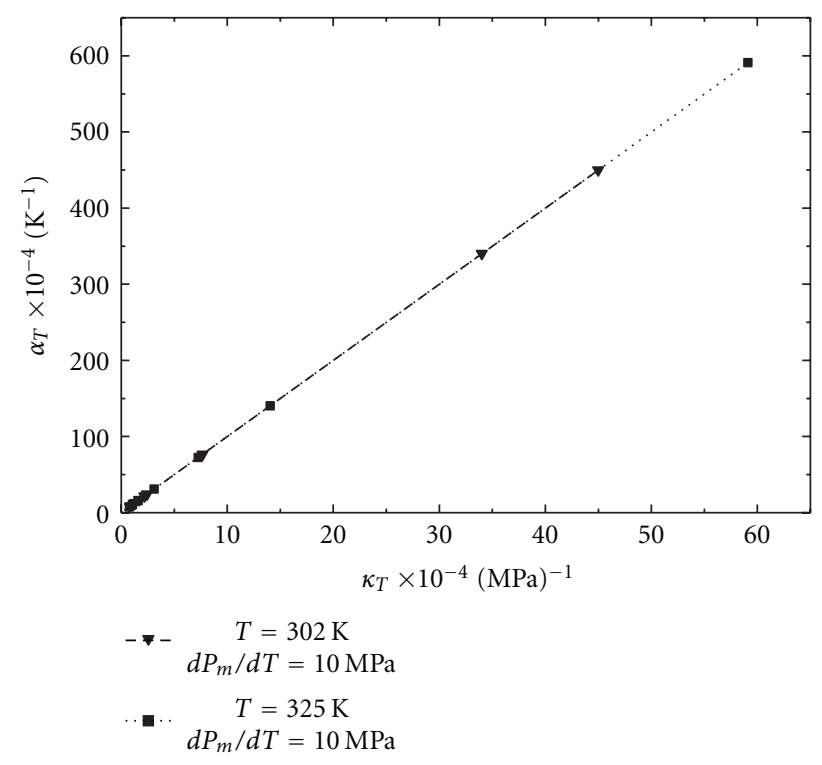

Figure 3: $\alpha_{p}$ versus $\kappa_{T}$ close to the premelting region of hexadecane at various $P$ for $P_{m}, T=302$ and $325 \mathrm{~K}$ (9). Observed $\alpha_{p}$ values [9] are shown here.

the critical exponents close to 2 [5]. Regarding the exponent value of 1.9 or 2, the melting zone can be shortened as the impurity decreases [19] close to the melting point in the premelting region of hexadecane. Our values of the critical exponent $\left(\gamma_{m}=2\right)$ for hexadecane can also be compared with those values for other organic compounds, which we obtained by Pruzan et al. [9]. Close to the melting point, they obtained the values of $\gamma_{m}=2$ (cyclohexane), 1.8 (carbon tetrachloride), 1.6 (benzene), and 1.5 (butanediol), as given in Table 2 in [5]. Similarly, the $\gamma_{m}$ values of 1.85 for hexamethylbenzene (HMB), 2 for paradichlorobenzene (PDCB), and the value of 1.7 for both paraozoxydianisol (PAA, C$\mathrm{N}$ transition) and heptyloxyazoxybenzene (HOAOB, C-S transition) compounds (Table 2 in [9]) can also be compared with our exponent value of $\gamma_{m}=2$ for hexadecane or $\gamma_{m}=1.9$ as obtained by Pruzan et al. [9]. The organic compounds indicated above have similar chemical structure (C-H- $\cdot$ bonds) within the close melting points and pressure ranges [9]. This shows that within the pressure ranges studied all those organic compounds exhibit similar divergence behaviour of the thermal expansion $\alpha_{p}$ near the melting point according to a power-law formula (2). Thus, our exponent value is within expectations in regard to the experimental results for various organic compounds stated above.

Our $\gamma$ values (Table 1) were used to calculate here the pressure dependence of volume $V_{T}(P)(6)$, isothermal compressibility $\kappa_{T}(4)$, and the specific heat $C_{p}(7)$, as indicated above. This calculation of $C_{p}$ and $\kappa_{T}$ then led us to plot $C_{p}$ against $\alpha_{p}$ (Figure 2) and $\alpha_{p}$ against $\kappa_{T}$ (Figure 3) with the zero values of the intercepts $T(d S / d T)_{m}$ and $(d V / d T)_{m} / V$, respectively, at the melting point in the premelting region of hexadecane. Those intercepts would have nonzero values 
according to the Pippard relations ((8) and (9)) if the temperature dependences of the entropy and volume are known experimentally or their functional forms can be derived for solid hexadecane. Values of the critical exponent $\gamma$ (Table 1) and also the slope $d P / d T$ were extracted from one source of experimental data [9] for hexadecane in the melting region. Regarding the anomalous behavior of $\alpha_{p}, \kappa_{T}$, and $C_{p}$ close to the melting point in the premelting region of hexadecane, the other sources of the experimental data rather than the measurements of the thermal expansivity $\alpha_{p}$ due to Pruzan et al. [9] can be used to examine our relations given here. Some new experimental measurements of the $\alpha_{p}, \kappa_{T}$, and $C_{p}$ at various pressures for constant temperatures can be performed in the premelting region near the melting point for hexadecane. When this experimental data is available in the literature which can be obtained to high accuracy, our analysis can then be reconsidered to examine the Pippard relations given here.

Regarding the uncertainties in the measurements of the thermal expansivity $\alpha_{p}$ for hexadecane, it was pointed out [9] that the uncertainty in the $\alpha_{p}$ data depends on the respective uncertainties in the heat of compression (1 or 2\%) and in the pressure step. It was also pointed out [9] that far from the transition lines, pressure steps around $20 \mathrm{MPa}$ caused an uncertainty in $\alpha_{p}$ of about $5 \%$. Close to the melting lines, within the pressure steps of $0.1-10 \mathrm{MPa}$, the uncertainty in $\alpha_{p}$ increased, whereas for the measurements of the molar volume $V$ the uncertainty was considerably small $(\sim 0.3 \%)$ [9].

Using the experimental data [9], we extracted that the uncertainties in $\alpha_{p}$ are about 5\% close to the melting point, which may be higher than this value, as pointed out by Pruzan et al. [9]. We represented the error bars in Figure 1 for $T=302 \mathrm{~K}$ and $325 \mathrm{~K}$, as stated above. This gave us the uncertainties in the critical exponent $\gamma$ for the thermal expansivity $\alpha_{p}$, which changes from 1.9 to 2.1 (Figure 1).

On the basis of the volume measured or calculated as a function of temperature in the premelting region of hexadecane, the temperature dependence of the thermal expansivity $\alpha_{p}$, isothermal compressibility $\kappa_{T}$, and the specific heat $C_{p}$ can be obtained, and the Pippard relations ((8) and (9)) can also be established at various temperatures near the melting point for constant pressures in this system.

\section{Conclusions}

We studied here the Pippard relations $\left(C_{p}\right.$ versus $\alpha_{p}$ and $\alpha_{p}$ versus $\kappa_{T}$ ) close to the melting point in the premelting region of hexadecane. Linear variations of $C_{p}$ with $\alpha_{p}$ (first Pippard relation) and $\alpha_{p}$ with $\kappa_{T}$ (second Pippard relation) were both obtained at various pressures for constant temperatures of $302 \mathrm{~K}$ and $325 \mathrm{~K}$ in this system.

The Pippard relations can be also be applied to the solid hexadecane in the premelting region using the temperature dependence of $C_{P}, \alpha_{P}$, and $\kappa_{T}$ for constant pressures. They can be verified for some other molecular organic compounds near the melting point when the experimental data for the thermodynamics quantities is available.

\section{Nomenclature}

$\alpha_{p}$ : Thermal expansivity

$\kappa_{T}$ : Isothermal compressibility

$C_{P}$ : Specific heat at constant pressure

$S: \quad$ Entropy

$V_{T}$ : Volume at constant temperature

$P_{m}$ : Melting pressure

$\gamma: \quad$ Critical exponent

A: Amplitude

$V_{m}$ : Melting volume

$P_{L}:$ Liquid pressure

$P_{C}$ : Critical pressure

$\gamma_{s}: \quad$ Critical exponent in the solid phase

$\gamma_{p}$ : Critical exponent in the precursor zone

$\gamma_{m}$ : Critical exponent in the melting zone.

\section{References}

[1] M. Milhet, J. Pauly, J. A. P. Coutinho, M. Dirand, and J. L. Daridon, "Liquid-solid equilibria under high pressure of tetradecane + pentadecane and tetradecane + hexadecane binary systems," Fluid Phase Equilibria, vol. 235, no. 2, pp. 173-181, 2005.

[2] B. He, V. Martin, and F. Setterwall, "Phase transition temperature ranges and storage density of paraffin wax phase change materials," Energy, vol. 29, no. 11, pp. 1785-1804, 2004.

[3] Y. Hayami and G. H. Findenegg, "Surface crystallization and phase transitions of the adsorbed film of $\mathrm{F}\left(\mathrm{CF}_{2}\right)_{12}\left(\mathrm{CH}_{2}\right)_{16} \mathrm{H}$ at the surface of liquid hexadecane," Langmuir, vol. 13, no. 18, pp. 4865-4869, 1997.

[4] S. Roke, J. Buitenhuis, J. C. van Miltenburg, M. Bonn, and A. Van Blaaderen, "Interface-solvent effects during colloidal phase transitions," Journal of Physics Condensed Matter, vol. 17, no. 45, pp. S3469-S3479, 2005.

[5] A. R. Ubbelohde, The Molten State of Matter, John Wiley and Sons, Chichester, UK, 1978.

[6] H. L. Finke, M. E. Gross, G. Waddington, and H. M. Huffman, "Low-temperature thermal data for the nine normal paraffin hydrocarbons from octane to hexadecane," Journal of the American Chemical Society, vol. 76, no. 2, pp. 333-341, 1954.

[7] M. Maroncelli, P. Q. Song, H. L. Strauss, and R. G. Snyder, "Nonplanar conformers and the phase behavior of solid $n$ alkanes," Journal of the American Chemical Society, vol. 104, no. 23, pp. 6237-6247, 1982.

[8] D. L. Dorset, B. Moss, J. C. Wittman, and B. Lotz, "The premelt phase of $n$-alkanes crystallographic evidence for a kinked chain structure," Proceedings of the National Academy of Sciences, vol. 81, no. 6, pp. 1913-1917, 1984.

[9] P. Pruzan, D. H. Liebenberg, and R. L. Mills, "Experimental evidence for a second-order transformation prior to melting in ammonia, organic compounds and ice I," Journal of Physics and Chemistry of Solids, vol. 47, no. 10, pp. 949-961, 1986.

[10] B. J. Bulkin and F. T. Prochaska, "Vibrational spectra of liquid crystals. II. The Raman spectrum of p-Azoxyanisole in Crystal, Nematic, and Isotropic Phases, $10-100-\mathrm{cm}^{-1}$ region," Journal of Chemical Physics, vol. 54, p. 635, 1971.

[11] R. Pynn and T. Riste, "Neutron scattering by a liquid crystal at temperatures close to its melting point," in Anharmonic Lattices, Structural Transitions and Melting, T. Riste, Ed., p. 363, Noordhoff, Leiden, The Netherlands, 1974. 
[12] R. Pynn, "Calculations of neutron diffraction patterns obtained with a nematic liquid crystal," Journal of Physics and Chemistry of Solids, vol. 34, no. 4, pp. 735-747, 1973.

[13] P. B. Miller and J. D. Axe, "Internal strain and Raman-active vibrations in solids," Physical Review, vol. 163, no. 3, pp. 924926, 1967.

[14] M. J. Buckingham and W. M. Fairbank, "The nature of the $\lambda$ transition in liquid Helium," in Progress in Low Temperature Physics, C. J. Gorter, Ed., p. 80, North-Holland, Amsterdam, The Netherlands, 1961.

[15] A. B. Pippard, The Elements of Classical Thermodynamics, Cambridge University Press, New York, NY, USA, 1957.

[16] J. Timmermans, Physico-Chemical Constant of Pure Organic Compounds, Binary Systems in Concentrated Solutions, vol. 4, Interscience Publishers, New York, NY, USA, 1960.

[17] R. Lipowsky, "Critical surface phenomena at first order bulk transitions," Physical Review Letters, vol. 49, pp. 1575-1578, 1982.

[18] R. Lipowsky and W. Speth, "Semi-infinite systems with firstorder bulk transitions," Physical Review B, vol. 28, no. 7, pp. 3983-3993, 1983.

[19] G. Pilcher, "A simplified calorimeter for the precise determination of purity," Analytica Chimica Acta, vol. 17, pp. 144-160, 1957. 

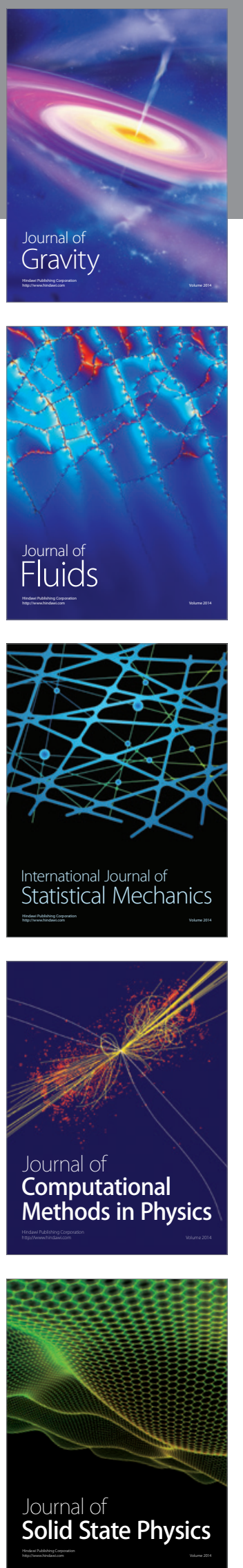

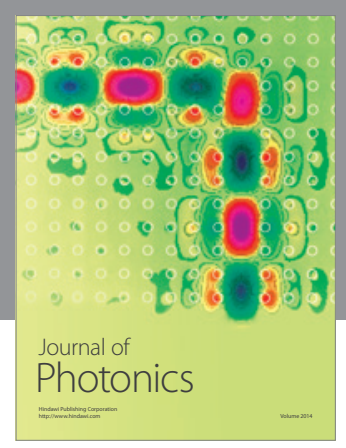

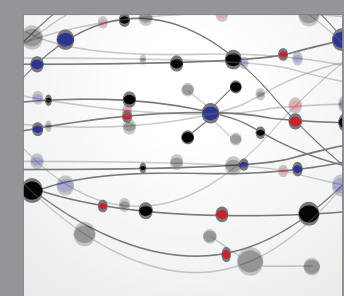

The Scientific World Journal
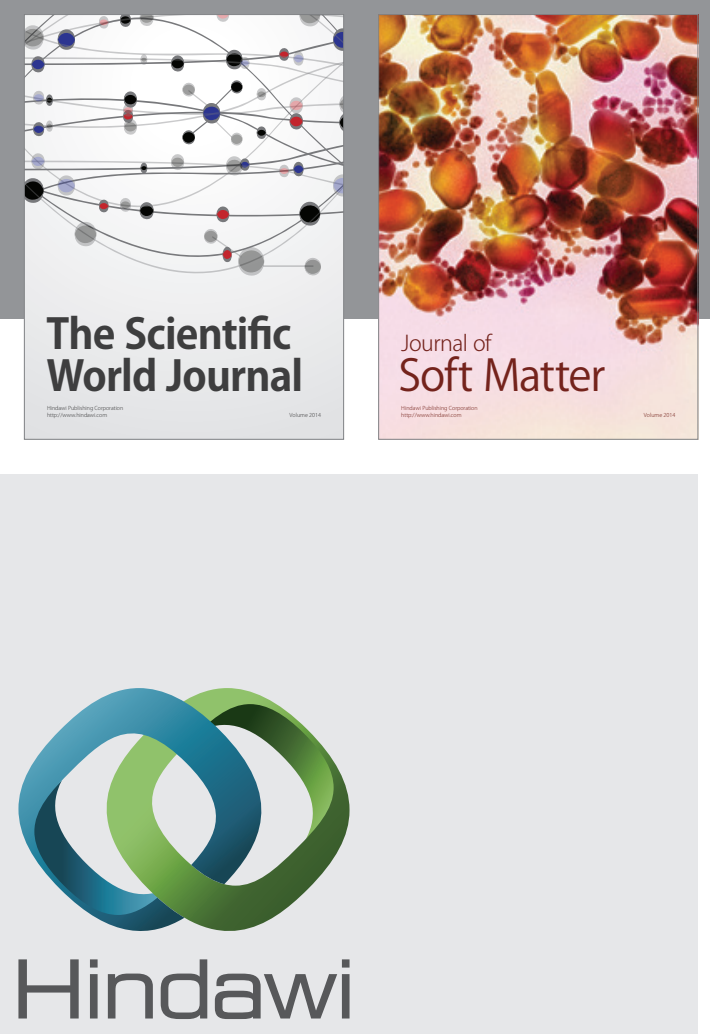

Submit your manuscripts at

http://www.hindawi.com
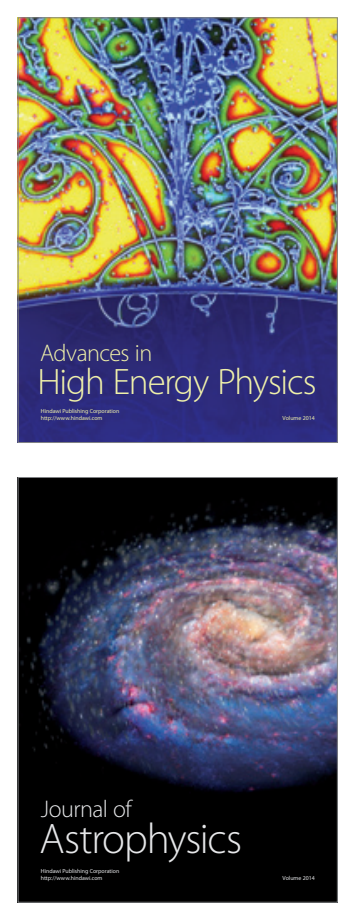
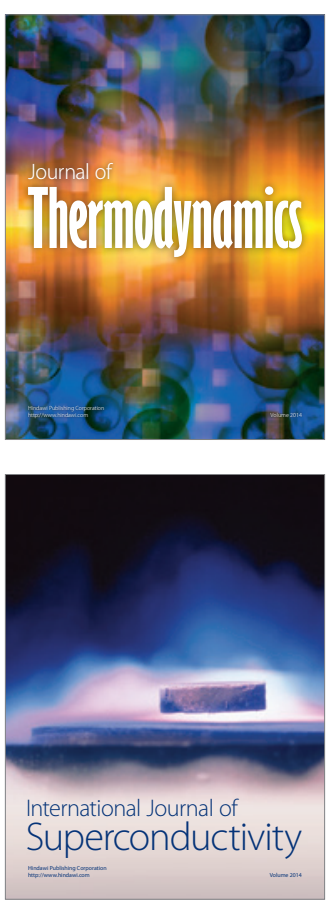
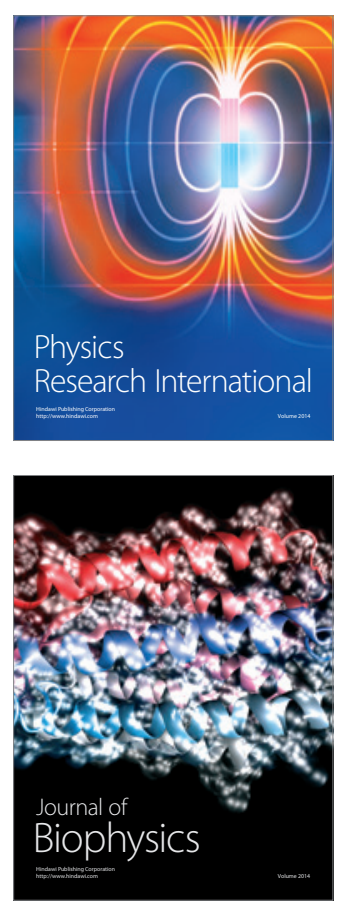
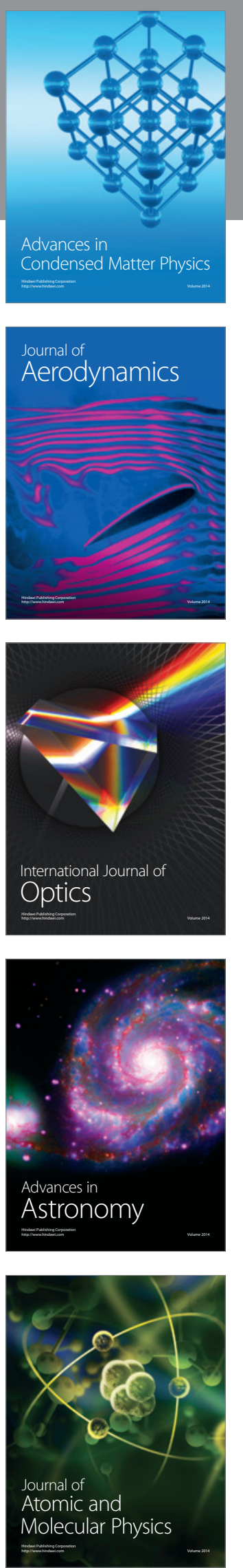